\title{
Supernova Rates
}

\section{By SIDNEY VAN DEN BERGH}

\author{
Dominion Astrophysical Observatory, National Research Council, 5071 West Saanich Road, \\ Victoria, British Columbia, V8X 4M6, Canada
}

Extragalactic supernova rates are reviewed. The main uncertainties in calculated rates are due to (1) the influence of the (still poorly known) luminosity function of supernova of a given type on "control times", to (2) uncertain corrections for possible inclination - dependent bias in supernova discovery probabilities, and (3) interstellar absorption. The total supernova rate in late-type galaxies is found to be $\sim 2\left(H_{o} / 75\right)^{2}$ supernovae (SNe) per century per $10^{10} \mathrm{~L}_{B}(\odot)$. This is consistent with the rate of $3 \mathrm{SNe}$ per century that is derived from the historical data on Galactic supernovae. It is, however, a source of some concern that none of the three Galactic $\mathrm{SNe}$ expected to have occurred during the last century was actually observed!

The expansion velocities of SNe Ia are found to correlate strongly with parent galaxy Hubble type. This relation is in the sense that low expansion velocities are only observed for those SNe Ia that occur in early-type galaxies. This suggests that $\mathrm{V}(\exp )$ correlates with the ages of $\mathrm{SNe}$ Ia progenitors. It is speculated that the progenitors of a few SNe Ia with high $V(\exp )$ values in $\mathrm{E}$ and $\mathrm{S} 0$ galaxies were formed during recent starbursts.

$\mathrm{SNe}$ Ia rates appear to be enhanced in post-starburst galaxies. It is suggested that supernova rates might be quite high in the recently discovered population of faint blue galaxies at intermediate redshifts.

\section{Extragalactic Supernova Rates}

The first estimate of extragalactic supernova rates was made by Zwicky (1938), who introduced the idea that "control time" was a critical factor needed to determine the supernova frequency. References to subsequent discussions of this topic are given in the reviews by van den Bergh (1991), van den Bergh \& Tammann (1991) and Tammann (1992). The most extensive databases on supernova discoveries, that have been analysed to derive supernova rates, are those based on the visual search program by Evans in Australia (Evans, van den Bergh \& McClure 1989) and on the Asiago and Crimean photographic surveys that have been analysed by Cappellaro et al. (1993ab). Supernova rates in early- type galaxies remain uncertain because of small-number statistics. In late-type galaxies the intrinsic supernova rates are poorly determined because inclinationdependent absorption corrections to these rates might be large (Tammann 1974, 1982, van den Bergh 1990, van den Bergh \& McClure 1990) and are poorly determined. In fact van den Bergh, McClure \& Evans (1987) and van den Bergh (1994) find no evidence for any significant inclination effect for the largest and most homogeneous data samples. Furthermore the discovery probabilities for supernovae are affected by galaxy surface brightness and by crowding. Additional uncertainties are introduced by night-to-night variations in the limiting magnitude of any particular search program. An interesting complication is provided by the fact that calculation of the control time for a specific species of supernova requires that the luminosity and lightcurve shape of that particular kind of supernova be known. Adopting a standard luminosity for all SNe Ia would, for example, overestimate the control time for subluminous objects such as SN $1991 \mathrm{bg}$ by an order of magnitude. Finally the relative numbers of supernovae of different types discovered in any particular survey will probably depend on the wavelength at which that search program was carried out. It is, for example, possible that the high frequency 
Table 1. Supernova Rates in $\mathrm{SNu}$

\begin{tabular}{clllll}
\hline \hline Galaxy type & Ia & Ib & II & source \\
\hline E-S0 & 0.15 & $\ldots$ & $\ldots$ & a \\
& 0.09 & $\ldots$ & $\ldots$ & b \\
& 0.13 & $\ldots$ & $\ldots$ & c \\
Sab,Sb & 0.15 & 0.3 & 0.9 & a \\
& 0.13 & 0.06 & 0.13 & b \\
& 0.17 & 0.13 & 0.30 & c \\
Sbc-Sd & 0.1 & 0.2 & 0.65 & a \\
& 0.18 & 0.08 & 0.43 & b \\
& 0.39 & 0.27 & 1.48 & c \\
\hline \hline
\end{tabular}

a observed by Evans et al. (1989)

b observed by Cappellaro et al. (1993b)

c Cappellaro et al. corrected for inclination and for surface brightness effects (Shaw 1979)

of SNe Ibc in the survey by Muller et al. (1992) is (at least in part) due to the fact that their unfiltered CCD detectors had a high red sensitivity, which favored discovery of strongly reddened supernovae with massive progenitors.

The supernova rates derived from the surveys of Evans et al. (1989) and of Cappellaro et al. (1993b) are listed in Table 1. The data in this table are for a Hubble parameter of $75 \mathrm{~km} \mathrm{~s}^{-1} \mathrm{Mpc}^{-1}$. For any other $\mathrm{H}_{o}$ value the entries in this table should be multiplied by $\left(\mathrm{H}_{o} / 75\right)^{2}$. The supernova rates in Table 1 are expressed in $\mathrm{SNu}[1 \mathrm{SNu}=$ one supernova per century per $\left.10^{10} \mathrm{~L}_{B}(\odot)\right]$. The supernova rates quoted by Evans et al. (1989) have been multiplied by a factor of 0.9 to convert them from the value $\mathrm{M}_{B}(\odot)=+5.37$ used in that paper to $\mathrm{M}_{B}(\odot)=+5.48$ adopted by Cappellaro et al. $(1993 \mathrm{~b})$. The differences between the rates of Evans et al. (1989) and of Cappellaro et al. (1993b) are due to (1) small number statistics, (2) absorption, surface brightness, and inclination corrections, and (3) differences in control times. To derive supernova rates from their observations Cappellaro et al. (1993b) apply the same surface brightness corrections (Shaw 1979) for all types of supernova. However, van den Bergh (1994) has recently shown that such corrections are small (or absent) for luminous SNe Ia, while they turn out to be quite large for SNe II which are, on average, much less luminous. The rate of SNe II in Sbc - Sd galaxies adopted by van den Bergh \& Tammann (1991) is 50\% higher than the fully corrected rate given in Table 1 . The reason for this difference is that Cappellaro et al. (1993ab) assume SNe II to be significantly brighter than do van den Bergh \& Tammann (1991). As a result Cappellaro et al. derive longer control times, and hence lower supernova frequencies. There is, however, also another reason why SNe Ibc and SNe II may be more frequent in late-type galaxies than Cappellaro et al. (1993b) find. According to current ideas (Branch, Nomoto \& Filippenko 1991, Van Dyk 1992) the progenitors of such supernovae are probably young massive stars. Many of such objects are likely to still be deeply embedded in the dusty clouds from which they were formed. [Van den Bergh (1992) finds that half of all nearby $\left(\mathrm{D}<3 \mathrm{kpc}\right.$ ) WR Stars have $\mathrm{A}_{V}$ $>7 \mathrm{mag}]$. This suggests that a substantial fraction of all supernovae in dust-rich latetype galaxies probably suffer so much absorption that they will not be discovered in most survey programs. This suspicion is strengthened by the observation (van den Bergh 1989) 
Table 2. Recent Galactic Supernovae

\begin{tabular}{llccl}
\hline \hline Name & Year & Distance (kpc) & Type \\
\hline RCW 86 & 185 & $0.95 \pm 0.4$ & II \\
Lupus & 1006 & $>1.9$ & Ia \\
Crab & 1054 & 2.0 & II \\
3C58 & $1181 ?$ & $2.6 \pm 0.2$ & II: \\
Tycho & 1572 & $3-4$ & Ia \\
Kepler & 1604 & $\geq 4.1 \pm 0.9$ & Ia? \\
Cas A & $1670:$ & 2.8 & Ibc \\
\hline \hline
\end{tabular}

that the frequency of supernovae in galaxies increases with increasing equivalent width of $\mathrm{H} \alpha+[\mathrm{NII}]$, but then suddenly appears to decrease in the (probably dusty) starburst galaxies with the largest $\mathrm{H} \alpha$ equivalent widths. Clearly it would be very important to check on this possibility by undertaking supernova searches at infrared wavelengths.

In view of all of the sources of error and bias discussed above extragalactic supernova rates are presently probably still uncertain by a factor of about two.

\section{The Galactic Supernova Rate}

The first modern discussion of the Galactic supernova rate is by Öpik (1953). He derived a rate of about one supernova per 300 years from the then known historical supernova remnants. However, Öpik already realized that the true supernova rate must be considerably higher than this because of interstellar extinction, and because the historical record of Galactic supernovae is incomplete. A bibliography listing recent estimates of the Galactic supernova frequency is given in van den Bergh \& Tammann (1991).

A compilation of data on supernovae that are known to have occurred in the Galaxy during the last two millennia is given in Table 2. The fact that this table lists only one object during the first millenium $(0-1000)$ and six during the second millenium $(1000-2000)$ strongly suggests that the most ancient historical data are also the most incomplete. A few additional ancient supernova suspects are listed by Wang et al. (1986).

More detailed information on the individual supernovae that are listed in Table 2 is given below:

- SN 185 - From x-ray observations of its remnant RCW 86 Kaastra et al. (1992) conclude that this object was a SN II that exploded at a distance of $0.95 \pm 0.4 \mathrm{kpc}$. Alternatively (Kaastra 1993) the progenitor might also have been a SN Ib embedded in a strong stellar wind.

- SN 1006 - The presence of Fe II absorption lines (Hamilton \& Fesen 1988) makes it virtually certain that this object was an SN Ia. From the width of the Balmer lines Smith et al. (1991) estimate a distance of $1.4-2.8 \mathrm{kpc}$. Recent HST observations by Wu et al. (1993) show that the Fe II lines have a width of $8300 \mathrm{~km} \mathrm{~s}^{-1}$. From this observation these authors conclude that the distance to the Lupus supernova is $>1.9$ $\mathrm{kpc}$. A distance greater than $3 \mathrm{kpc}$ would, however, make the luminosity of SN 1006 excessive.

- SN 1054 - The Crab nebula contains Balmer line emitting filaments. It must therefore, by definition, be a SN II. The low expansion velocity of the Crab also excludes the 
possibility that it might have been a SN Ia. The distance to the Crab is estimated to be $2.0 \mathrm{kpc}$ (Woltjer 1958, Trimble 1968). In their review Davidson \& Fesen (1985) place the Crab nebula at a distance of between 1.5 and $2.2 \mathrm{kpc}$.

- SN 1181 - The probable association of this object with 3C58 is discussed in Stephenson (1971) and Clark \& Stephenson (1977), but see Becker, Helfand \& Szymodiak (1982) for a contrary opinion. The similarity of the radio remnant of $3 \mathrm{C} 58$ (Wilson \& Weiler 1976) to that of the Crab makes it highly probable that the progenitor of SN 1181 was also a SN II. Green \& Gull (1982) estimate the distance to $3 \mathrm{C} 58$ to be $2.6 \mathrm{kpc}$, with an (optimistic?) error of $\pm 0.2 \mathrm{kpc}$.

- SN 1572 - Distance determinations to the remnant of Tycho's supernova (3C10) have been reviewed by de Vaucouleurs (1985). From 18 rather discordant distance estimates de Vaucouleurs concludes that its distance is $\mathrm{D}=3.2 \pm 0.3 \mathrm{kpc}$. More recently Strom (1988) obtained a distance of $2.5 \mathrm{kpc}$. However, Strom also finds $\mathrm{D}$ (Tycho)/D(Lupus) = 1.74. With $\mathrm{D}$ (Lupus) $>1.9 \mathrm{kpc}$ (Wu et al. 1993) this would yield $\mathrm{D}$ (Tycho) $>4.4 \mathrm{kpc}$. A smaller distance of $1.5-3.1 \mathrm{kpc}$ has been estimated from the width of the Balmer lies by Smith et al. (1991). A distance of $3-4 \mathrm{kpc}$ will be tentatively adopted. Following Weiler \& Sramek (1988) it will be assumed that SN 1572 was a supernova of type Ia.

- SN 1604 - Braun (1987) finds a distance of $4.1 \pm 0.9 \mathrm{kpc}$ for the remnant of Kepler's supernova. Using similar techniques he also finds $\mathrm{D}=2.3 \mathrm{kpc}$ for the remnant of Tycho's supernova. This suggests that the true distance to SN 1604 might actually be somewhat larger than $4.1 \mathrm{kpc}$. The nature of the progenitor of SN 1604 remains in dispute. Bandiera (1987) has suggested that it was a massive runaway star that originated in the Galactic disk. However, it is difficult to obtain the high space velocity of the remnant $(278 \pm 12$ $\mathrm{km} \mathrm{s}^{-1}$ ) found by Bandiera \& van den Bergh (1991) by ejection from a young starcluster (Leonard \& Duncan 1988) or by disruption of a massive binary system (Leonard \& Dewey 1993). This suggests that the progenitor of SN 1604 is more likely to have been a high-velocity halo star of Population II.

- SN 1670: - The absence of hydrogen from the bulk of the fast-moving ejecta of Cassiopeia A suggests that the progenitor of this object was a SN Ibc. However, detection of a few high-velocity knots emitting $\mathrm{H} \alpha$ and [NII] in the outermost part of the remnant of Cas A (Fesen, Becker \& Blair 1987) indicates that the progenitor might have had a thin outer hydrogen layer (Fesen, Becker \& Goodrich 1988). Possibly it was a late WN star. Intercomparison of the radial velocities and proper motions in the remnant of Cas A yield an uncertain distance of $2.8 \mathrm{kpc}$.

Taken at face value the data in Table 2 suggest that the total supernova rate within $4 \mathrm{kpc}$ of the sun is three per millennium. By the same token the number of SN Ibc and SN II, which have massive progenitors, and that are located within $4 \mathrm{kpc}$, is 2 per millennium. A slightly higher rate of three core-collapse SNe per millenium would be derived using only those supernovae that are known to have occurred within $4 \mathrm{kpc}$ during the Second Millennium.

From a rate of 3 supernovae per millenium within $4 \mathrm{kpc}$, and an assumed thickness of the disk of $0.3 \mathrm{kpc}$, one finds a local supernova rate of $2 \times 10^{-4} \mathrm{SNe} \mathrm{kpc}^{-3} \mathrm{yr}^{-1}$. For an assumed disk age of $10 \mathrm{Gyr}$ it then follows that the total number of supernovae that have exploded in the solar neighborhood is $2 \times 10^{6} \mathrm{kpc}^{-3}$. On average the nearest supernova would therefore have occurred at a distance of $\sim 5 \mathrm{pc}$. Such a supernova would have had $\mathrm{V} \sim-20$, which is bright enough to cause some evaporation of gas from the surfaces of comets in the Oort cloud, and perhaps destruction of the Earth's ozone layer (Ellis \& Schramm 1993).

SNe with massive progenitors are probably distributed in the Galaxy in much the same fashion as are stars of Population I. Following Ratnatunga \& van den Bergh (1989) it will 
be assumed that these objects exhibit an exponential radial density distribution with a scale-length of $4 \mathrm{kpc}$ that extends from $3 \mathrm{kpc}$ to $15 \mathrm{kpc}$. With such a distribution $\sim 9 \%$ of all Galactic OB stars will be located within $4 \mathrm{kpc}$ of the Sun, which is assumed to have $\mathrm{R}_{o}=8.5 \mathrm{kpc}$. If $9 \%$ of all supernovae with massive progenitors also occur within $4 \mathrm{kpc}$ of the Sun, then the total rate of Galactic supernovae with massive progenitors is $2 / 0.09=22$ (or $3 / 0.09=33$ ) per millennium. (Including SNe Ia would increase the Galactic supernova rate by $20-25 \%$ ). It is noted in passing that the Galactic corecollapse supernova rate of $2-3$ per century, that was derived above, is marginally higher than the rate of $1.0_{-0.6}^{+1.5}$ per century which Ratnatunga \& van den Bergh (1989) derived from the Scalo (1986) luminosity function and the assumption that the progenitors of core-collapse supernovae have $\mathbf{M}>8 \mathrm{M} \odot$. The constraint on the rate of core-collapse supernovae, that is provided by the Scalo (1986) luminosity function, might be eased somewhat if some SNe Ibc are formed by mass transfer in binaries with individual main sequence components which have masses that are slightly smaller than $8 \mathrm{M} \odot$.

From a review of all Galactic luminosity indicators van den Bergh (1988) concluded that the Milky Way System has $\mathrm{L}_{B}=(2.3 \pm 0.6) \times 10^{10} \mathrm{~L}_{B}(\odot)$. This value of the Galactic luminosity, when combined with a supernova rate of 3 per century, yields a Galactic rate of $1.3 \mathrm{SNu}$ for supernovae with massive progenitors. This value is in reasonable agreement with the total ( $\mathrm{SNe} \mathrm{Ib}+\mathrm{SNe}$ II) rate of $1.75 \mathrm{SNu}$ for late-type galaxies given in Table 1 .

In the Galactic model of Ratnatunga \& van den Bergh (1989) $0.5 \%$ of all star formation taken place within one kpc of the Sun. For a total Galactic SN Ib + SN II rate of 3 per century the a priori probability of observing a supernova with a massive progenitor at $D$ $\leq 1.0 \mathrm{kpc}$ during the last 20 centuries is therefore $3 \times 20 \times 0.005=0.3$. The occurrence of SN 185 at a distance of only $950 \mathrm{pc}$ (Kaastra et al. 1992) is therefore not unreasonable. It is, however, a source of some concern (Poisson statistics give $\mathrm{p} \simeq 0.05$ ) that none of the three Galactic supernovae expected to have occurred during the past century was actually observed! Virginia Trimble has, however, reminded me of the remote possibility that a heavily reddened faint Galactic supernova might have been cataloged as a common nova.

\section{The Expansion Velocities of Supernovae of Type Ia}

Elliptical galaxies consist mainly of old stars, whereas spiral galaxies contain stellar populations with a wide range in ages. If the characteristics of SNe Ia depend on the ages of their progenitors then supernovae of type Ia in ellipticals should exhibit systematic differences from those that occur in spirals. Recently van den Bergh \& Pazder (1992) found that $\mathrm{SNe}$ Ia in spirals were, on average, $\sim 1$ mag more luminous than those in ellipticals. However, van den Bergh \& Pierce (1992) show that this conclusion depends critically on the values for the intrinsic colors that are assumed for SNe Ia. Such uncertainties are avoided in spectroscopic studies of supernovae.

In a recent paper Branch \& van den Bergh (1993) have shown that the expansion velocities of SNe Ia appear to correlate with Hubble type, in the sense that supernovae in early-type galaxies tend to have lower expansion velocities than do those that occur in late-type galaxies. All seven $\mathrm{SNe}$ Ia with expansion velocities at 10 days past maximum $\mathrm{V}(10)<9500 \mathrm{~km} \mathrm{~s}^{-1}$ occurred in early-type (E,S0,Sa) galaxies. On the other hand only two out of $26(12 \%)$ of the SNe Ia with $\mathrm{V}(10) \geq 9500 \mathrm{~km} \mathrm{~s}^{-1}$ occurred in E - Sa galaxies. Taken at face value this observation suggests that $\mathrm{SNe}$ Ia with low expansion velocities may belong to an older stellar population than do those with high expansion velocities. This conclusion is consistent with the observation that the SNe Ia in elliptical galaxies 


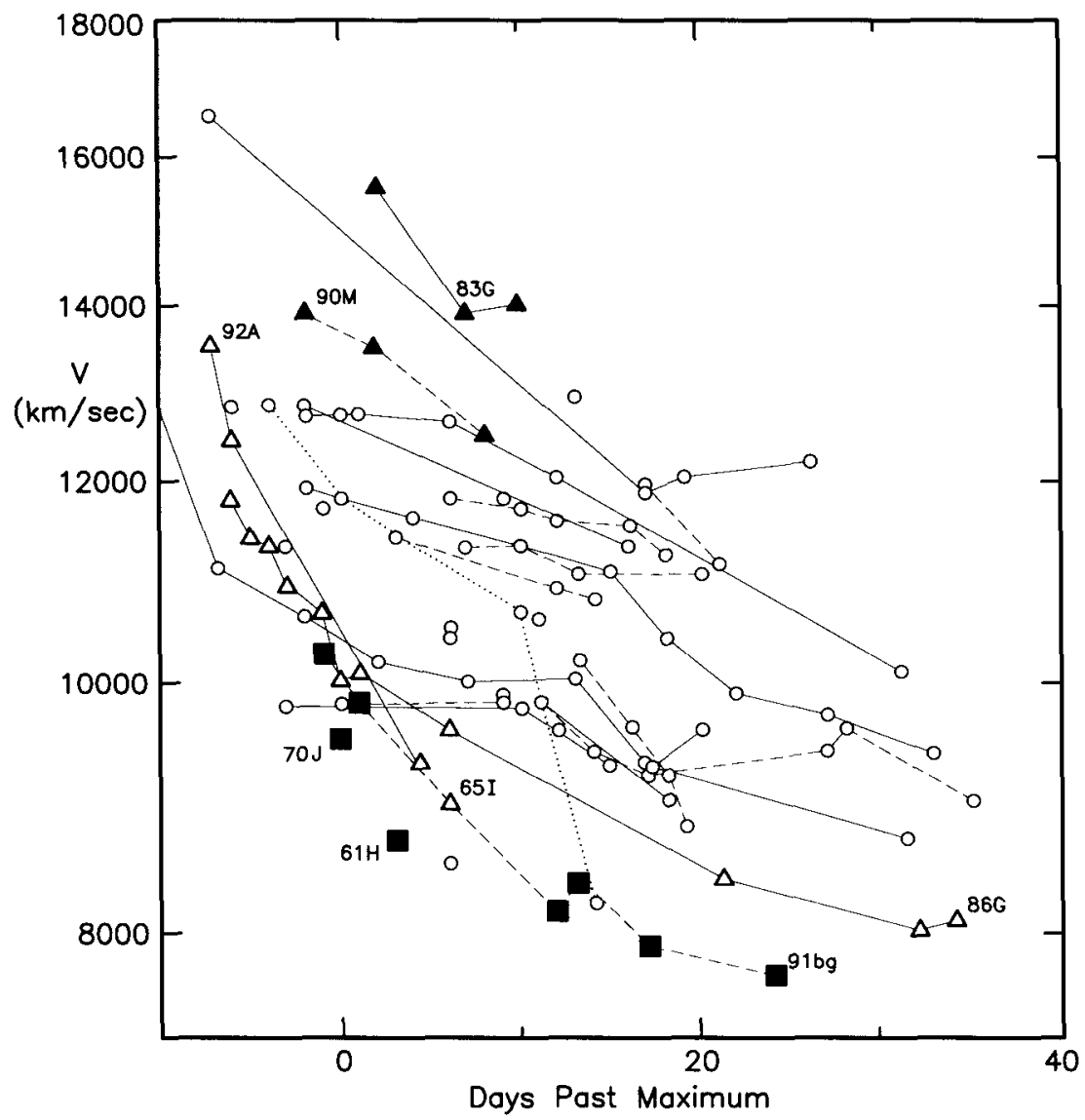

Figure 1. Expansion velocity versus time for supernovae of type Ia. [Adapted from Branch \& van den Bergh (1993)]. Points joined by thin lines belong to a single supernova. Note that the $\mathrm{SNe}$ Ia in elliptical galaxies (squares) fall below those that occur in other types of galaxies.

all had particularly low velocities of expansion at 10 days past maximum: $8300 \mathrm{~km} \mathrm{~s}^{-1}$ for SN $1961 \mathrm{H}$ in NGC 4564, and $8500 \mathrm{~km} \mathrm{~s}^{-1}$ for SN $1991 \mathrm{bg}$ in NGC 4374, and 8700 $\mathrm{km} \mathrm{s}^{-1}$ for SN $1970 \mathrm{~J}$ in NGC 7619. Fig. 1 shows that the SNe Ia in elliptical galaxies lie along the lower boundary of the observed V(exp) versus expansion age distribution.

The only known exceptions to the rule that $\mathrm{SNe} \mathrm{Ia}$ in early-type galaxies have low expansion velocities are SN $1983 \mathrm{G}$ in $\mathrm{NGC} 4753\left[\mathrm{~V}(10)=13600 \mathrm{~km} \mathrm{~s}^{-1}\right]$, and SN $1990 \mathrm{M}$ in NGC $5493\left[\mathrm{~V}(10)=12220 \mathrm{~km} \mathrm{~s}^{-1}\right]$. Véron \& Véron-Cetty (1985) note that NGC 5493 is one of the three bluest objects in the sample of 69 giant E and S0 galaxies for which they obtained UBV photometry. This result suggests that NGC 5493 contains a sizable young or intermediate-age population with which SN $1990 \mathrm{M}$ might have been associated. From the structure of the dust lanes in the very peculiar S0 galaxy NGC 4753 Steiman-Cameron, Kormendy \& Durisen (1992) conclude that this galaxy captured a gas- and dust-rich dwarf $(0.5-1.0)$ Gyr ago. Possibly the progenitor of the very fastexpanding SN $1983 \mathrm{G}$ was a member of the intermediate-age population of this dwarf, or of the star burst population associated with its capture.

It has been noted previously (Oemler \& Tinsley 1979, Thompson 1981, van den Bergh \& Tammann 1991) that the frequency of SNe Ia appears to be particularly high in 
amorphous (Sandage \& Brucato 1979) galaxies, which are a subset of the galaxies referred to as I0 by de Vaucouleurs (1959). On deep exposures many of these galaxies show the light distribution that is characteristic of elliptical galaxies (Caldwell 1982). This suggests (Véron \& Véron-Cetty 1985) that such objects are $\mathrm{E}$ or $\mathrm{S} 0$ galaxies in which the true underlying morphology is veiled by dust and overshadowed by the afterglow of a violent burst of star formation that took place in the relatively recent past. Van den Bergh (1980) has, for example, proposed that the amorphous galaxy NGC 5253 experienced a violent burst of star formation after it captured gas from M83 between 0.1 Gyr and 1.0 Gyr ago. In particular he suggested that the occurrence of two SN Ia (1895B and 1972E) in this relatively faint object during the past century was due to the high rate of star formation in this galaxy in the recent past. The fact that the expansion velocity of SN $1972 \mathrm{E}\left[\mathrm{V}(10)=10700 \mathrm{~km} \mathrm{~s}^{-1}\right]$ was not low is consistent with the hypothesis that only those $\mathrm{SNe}$ Ia with low expansion velocities are produced by progenitors that belong to an old stellar population. If the speculations outlined above are correct then a high rate of SNe Ia with large expansion velocities is diagnostic of objects that have experienced a starburst in their recent past (van den Bergh 1993).

\section{Supernovae in Distant Blue Galaxies}

Recently a number of investigations (Broadhurst, Ellis \& Shanks 1988, Colless et al. 1990, Lilly, Cowie \& Gardner 1991) have found that the number of faint blue galaxies grows more rapidly than expected with increasing lookback time. The number of such objects appears to be 3 to 5 times greater than would be expected from linear extrapolation of the present population of galaxies under the assumption of no evolution (Cowie, Songaila \& $\mathrm{Hu} 1991)$. At $\mathrm{B} \sim 23$ these blue galaxies are typically observed to have redshift $\mathrm{z} \sim 0.25$ and a median luminosity $\mathrm{M}_{B} \simeq-17.5\left(\mathrm{H}_{o}=75 \mathrm{~km} \mathrm{~s}^{-1} \mathrm{Mpc}^{-1}\right.$ assumed $)$. A magnitude-selected sample of such faint blue galaxies therefore comprises objects which typically have luminosities comparable to that of the Large Magellanic Cloud (LMC).

There are a number of reasons for suspecting that the blue galaxies at $\mathrm{z} \sim 0.25$ might exhibit a high supernova rate:

(a) Many of these objects are probably experiencing starbursts at the present time. They should therefore be producing SNe Ibc and SNe II (which have massive progenitors) at a high rate. Furthermore both theory (Greggio \& Renzini 1983) and observations of NGC 5253, which has produced two SNe I during the last century, suggest (van den Bergh 1980) that the SN Ia rate may be greatly enhanced a few hundred million years after a starburst.

(b) Supernova in distant blue (dwarf) galaxies will typically have $\mathrm{M}(\max )$ values that are brighter than, or comparable to, those of their parent galaxies. This should facilitate the discovery of such supernovae.

(c) If the blue galaxies at intermediate redshifts are currently undergoing bursts of star formation then their equilibrium (post-starburst) luminosities will be quite low. Such intrinsically faint galaxies are known (van den Bergh \& Pierce 1990) to have a low dust content. Their supernovae will therefore not be significantly dimmed by interstellar absorption.

A lower limit to the supernova frequency in faint blue galaxies at $\mathrm{B} \sim 23$ can be obtained by assuming that these objects are similar to nearby galaxies of types Sbc - Sd. Table 1 suggests that such galaxies have supernova rates of 1 to $2 \mathrm{SNu}$.

Note that time dilation will increase the discovery probability of distant supernova because their lightcurves are stretched by a factor of $1+\mathrm{z}$. However, this advantage is nullified by a change in the supernova rates by a factor of $(1+z)^{-1}$. A search for 
supernovae at cosmological distances has recently been started by Couch et al. (1992). After maximum light some supernova spectra are observed to contain relatively little energy at $\lambda<4000 \AA$. This suggests that searches for supernovae with $z<0.5$ are best carried out in the $\mathrm{R}$ passband. For nearer galaxies searches for supernovae in the red (Muller et al. 1992) will also benefit from lower absorption at longer wavelengths.

\section{Acknowledgements}

I thank Drs. Cappellaro, Fesen, Hamilton, Strom, Trimble, Weiler and Wu for helpful comments, preprints and (or) suggestions.

\section{R EFERENCES}

Bandiera, R. 1987, ApJ, 319, 885

Bandiera, R., \& van den Bergh, S. 1991, ApJ, 374, 186

Becker, R.H., Helfand, D.J., \& Szymowiak, A.E. 1982, ApJ, 255, 557

Branch, D., Nomoto, K., \& Filippenko, A.V. 1991 Comments Astrophys. 15, 221

Branch, D., \& van den Bergh, S. 1993, AJ, 105, 2231

Braun, R., 1987 A\&A, 171, 233

Broadhurst, T.J., Ellis, R.S., \& Shanks, T. 1988, MNRAS, 235, 827

Caldwell, C.N. 1982, unpublished Ph.D. Thesis, Yale University

Cappellaro, E., Turatto, M., Benetti, S., Tsvetkov, D.Yu., Bartunov, O.S., \& Makarova, I.N. 1993a, A\&A, 268, 472

Cappellaro, E., Turatto, M., Benetti, S., Tsvetkov, D.Yu., Bartunov, O.S., \& Markarova, I.N. $1993 \mathrm{~b}, \mathrm{~A} \& \mathrm{~A}, 273,383$

Clark, D.H. \& Stephenson, F.R. 1977, The Historical Supernovae, (Oxford: Pergamon) pp.161171

Colless, M., Ellis, R.S., Taylor, K., \& Hook, R.N. 1990, MNRAS, 244, 408

Couch, W.J., Perlmutter, S., Newburg, H.J.M., Pennypacker, C., Goldhaber, G., Muller, R., \& Boyle, B.J. 1992, Proc. Astron. Soc. Australia, 9, 261

Cowie, L.L., Songaila, A., \& Hu, E.M. 1991, Nature, 354, 460

Davidson, K., \& Fesen, R.A. 1985, ARAA, 23, 119

de Vaucouleurs, G. 1959, Handb. d. Physik, 53, 275

de Vaucouleurs, G. 1985, ApJ, 289, 5

Ellis, J., \& Schramm, D.N. 1993, preprint

Evans, R., van den Bergh, S., \& McClure, R.D. 1989, ApJ, 345, 752

Fesen, R.A., Becker, R.H. \& Blair, W.P. 1987, ApJ, 313, 378

Fesen, R.A., Becker, R.H., \& Goodrich, R.W. 1988, ApJ, 329, L89

Green, D.A., \& Gull, S.F., 1982, Nature, 299, 606

Greggio, L., \& Renzini, A. 1983, A\&A, 118, 217

Hamilton, A.J.S., \& Fesen, R.A. 1988, ApJ, 327, 178

Kaastra, J.S., 1993, private communication

Kaastra, J.S., Asaoka, I., Koyama, K., \& Yamauchi, S. 1992, A\&A, 264, 654

Leonard, P.J.T., \& Dewey, R.J. 1993, BAAS, 24, 1177

Leonard, P.J.T., \& Duncan, M.J. 1988, AJ, 96, 222

Lilly, S.J., Cowie, L.L., \& Gardner, J.P. 1991, ApJ, 369, 79

Muller, R.A., Newberg, H.J.M., Pennypacker, C.R., Perlmutter, S., Sasseen, T.P., \& Smith, C.K. 1992, ApJ, 384, L9 
Oemler, A., \& Tinsley, B.M. 1979, A.J, 84, 985

Öpik, E. 1953, Irish A.J., 2, 219

Ratnatunga, K.U., \& van den Bergh, S. 1989, ApJ, 343, 713

Sandage, A., \& Brucato, R. 1979, AJ, 84, 472

Scalo, J.M. 1986, Fund. Cosmic Phys. 11, 1

Shaw, R.L. 1979, A\&A, 76, 188

Smith, C.R., Kirsher, R.P., Blair, W.P., \& Winkler, P.F. 1991, ApJ, 375, 650

Steiman-Cameron, T.Y., Kormendy, J., \& Durisen, R.H. 1992, AJ, 104, 1339

Stephenson, F.R. 1971, QJRAS, 12, 10

Strom, R.G., 1988, MNRAS, 230, 331

Tammann, G.A. 1974 in Supernovae and Supernova Remnants ed. C.B. Cosmovici (Dordrecht: Reidel) p.155

Tammann, G.A. 1982 in Supernovae: A Survey of Current Research eds. M.J. Rees and R.J. Stonehouse (Dordrecht: Reidel) p.371

Tammann, G.A. 1992, preprint of Les Houches Summer Course Lecture

Thompson, L.A. 1981, PASP, 93, 176

Trimble, V. 1968, AJ, 73, 535

van den Bergh, S. 1971, ApJ, 165, 457

van den Bergh, S. 1980, PASP, 92, 122

van den Bergh, S., 1988, Comments Astrophys., 12, 131

van den Bergh, S., 1989, AJ, 101, 845

van den Bergh, S., 1990, A\&A, 231, L27

van den Bergh, S. 1991, Physics Reports, 204, 386

van den Bergh, S. 1992, ApJ, 390, 133

van den Bergh, S. 1993, in preparation

van den Bergh, S. 1994, in preparation

van den Bergh, S., \& McClure, R.D. 1990, ApJ, 359, 277

van den Bergh, S., McClure, R.D., \& Evans, R. 1987, ApJ, 323, 44

van den Bergh, S., \& Pazder, J. 1992, ApJ, 390, 34

van den Bergh, S., \& Pierce, M.J. 1990, ApJ, 364, 444

van den Bergh, S., \& Pierce, M.J. 1992, PASP, 104, 408

van den Bergh, S., \& Tammann, G.A. 1991, ARAA, 29, 363

Van Dyk, S.D. 1992, AJ, 103, 1788

Véron, P., \& Véron-Cetty, M.-P. 1985, A\&A, 145, 433

Wang, Z.R., Liu, J.Y., Gorenstein, P., \& Zombeck, M.V. 1986 in Highlights of Astronomy, ed. J.-P. Swings (Dordrecht: Reidel) p. 583

Weiler, K.W., \& Sramek, R.A. 1988, ARAA, 26, 295

Wilson, A.S., \& Weiler, K.W. 1976, A\&A, 53, 89

Woltjer, L. 1958, Bull. Astr. Inst. Netherlands, 14, 39

Wu, C.C., Crenshaw, D.M., Fesen, R.A., Hamilton, A.J.S., \& Sarazin, C.L. 1993, ApJ, 416, 27

Zwicky, F. 1938, ApJ, 88, 529 